\title{
Frequence Conversion for RGB Light Sources
}

\author{
Yongyuan ZHU \\ National Laboratory of Solid State Microstructures, Nanjing University, Nanjing 210093, China
}

(Received January 13, 2005)

\begin{abstract}
Red, green, and blue, the three primary colors, are essential for laser-based color displays that take advantage of the high brightness, spectral purity and extremely large depth of focus of laser sources. There remains a problem as how to generate laser radiation in these colors in a single compact laser system in order to reduce cost, size and to obtain potential integration. Recently many attempts have been made towards this goal. In the as-grown crystals of $\mathrm{LiNbO}_{3}$ and $\mathrm{SBN}$, the domain structures are formed spontaneously during the crystal growth process, resulting in very low conversion efficiency. Our study showed that with a careful design of the domain engineering, efficient RGB light generateon can be realized by coupled quasi-phase matching method. A brief review will be given in this field.
\end{abstract}

Key Words: Frequency conversion, Ferroelectric domain engineering, Quasi-phase-matching, RGB light source

\section{Introduction}

With the development of modern optics technology, laser sources with wider range of wavelengths are needed more frequently. Optical frequency conversion with nonlinear optical materials is a key means by which such sources can be achieved. Thus this field has attracted much interest in recent years. For example, red, green, and blue, the three primary colors, are essential for laser-based color displays that take advantage of the high brightness, spectral purity and extremely large depth of focus of laser sources. ${ }^{1)}$ There remains a problem as how to generate laser radiation in these colors in a single compact laser system in order to reduce cost, size and to obtain potential integration. In the past, many attempts at simultaneous generation of RGB light have been made. Some progress was made in waveguides; for example, observations of quasi-phase-matching (QPM) of RGB lights using $\mathrm{LiNbO}_{3}$ waveguides ${ }^{2,3)}$ and using a dye-doped polymer waveguide ${ }^{4)}$ were reported. For bulk samples, results were obtained by several different schemes, including self-frequency mixing with $\mathrm{Nd}^{3+}$-doped nonlinear crystals, ${ }^{5-7)}$ multiple and coupled quasi-phase-matched interactions from domain-engineered crystals. ${ }^{8-16)}$ However, the output power attained was comparatively low. Thus the simultaneous generation of the three primary colours is still a challenge. Here we shall give a brief review of the work done in this field with the bulk materials.

\section{Self-frequency mixing with $\mathrm{Nd}^{3+}$-doped nonlinear crystals}

It is advantageous to generate laser radiation in RGB colors in a single compact laser system in order to reduce cost, size and to obtain potential integration. Diode-pumped infrared solid state lasers based on $\mathrm{Nd}^{3+}$ ion provide an excellent possibility to develop such devices when combined with nonlinear frequency conversion to the visible. In particular, nonlinear crystals doped with $\mathrm{Nd}^{3+}$ ions can be pumped efficiently by GaAlAs laser di- odes emitting around 800 and $750 \mathrm{~nm}$ due to the typical intense absorption which $\mathrm{Nd}^{3+}$ ions present in these bands. Laser action around 900, 1060, and $1300 \mathrm{~nm}$ has been demonstrated in a variety of $\mathrm{Nd}^{3+}$-doped laser crystals, corresponding to optical transitions from the metastable state $\left({ }^{4} \mathrm{~F}_{3 / 2}\right)$ to lower stark states ${ }^{4} \mathrm{I}_{9 / 2},{ }^{4} \mathrm{I}_{11 / 2}$ and ${ }^{4} \mathrm{I}_{13 / 2}$, respectively. ${ }^{5-7)}$

$\mathrm{Nd}: \mathrm{YAl}_{3}\left(\mathrm{BO}_{3}\right)_{4}(\mathrm{NYAB})$ is a nonlinear crystal characterized by high damage resistance, high segregation coefficient for $\mathrm{Nd}^{3+}$ ions, absence of photorefractive damage, low laser thresholds for laser oscillation at 1 and $1.3 \mu \mathrm{m}$ and excellent transmission for the expected visible wavelengths. Its nonlinear properties are mainly due to the two $\mathrm{BO}$ groups, being one in the (001) plane and another one slightly tilted with respect to this plane. All these properties ensure that NYAB is an efficient infrared laser system under both low and high pump power densities. In addition, NYAB has relatively high nonlinear coefficient and its dispersion relations for the refractive indexes allows for simultaneous generation of second harmonic generation (SHG) and frequency sum mixing processes.

In Frequency conversion, two conservation laws should be fulfilled: one is the energy conservation and the other momentum conservation. Taking SHG as an example, these two conservation laws can be expressed as

$$
\begin{aligned}
& \omega_{2}=\omega_{1}+\omega_{1} \\
& k_{2}=2 k_{1}
\end{aligned}
$$

Here $\omega_{i}$ and $k_{i}$ are the angular frequency and wavevector, respectively (the subscript 1 for the fundamental and 2 for the second harmonic).

Continuous RGB generation was experimentally demonstrated from a NYAB by using the birefringence phase matching (BPM) technique. Red $(669 \mathrm{~nm})$, green $(503 \mathrm{~nm})$ and blue $(481 \mathrm{~nm})$ were generated from the same $\mathrm{Nd}^{3+}$ doped nonlinear crystal. The crystal was either end-pumped by using a 700-850 nm tunable argon pumped Ti:sapphire laser or end-pumped by using a laser diode $(807 \mathrm{~nm})$ and a Ti:Sapphire laser $(750 \mathrm{~nm})$ simultaneously. 
In the former case, the red, green and blue are not generated simultaneously. While the latter case increases the complexity of the system. The laser radiation of $\mathrm{Nd}^{3+}$ ions at $1338 \mathrm{~nm}$ $\left({ }^{4} \mathrm{~F}_{3 / 2} \rightarrow{ }^{4} \mathrm{I}_{13 / 2}\right.$ channel) was the fundamental wave in this configuration. The red at $669 \mathrm{~nm}$ was obtained by self-frequencydoubling (SFD) of the fundamental wave, the green at $505 \mathrm{~nm}$ and the blue at $481 \mathrm{~nm}$ were obtained by self-frequency-mixing (SFM) of the fundamental wave and the pump radiation at 807 and $755 \mathrm{~nm}$, respectively. For a total pump power of $1.2 \mathrm{~W}$ (600 $\mathrm{mW}$ from both Ti:Sapphire and laser diode), the powers of green and blue laser obtained are of the order of microwatts, whilst the power of red laser generated is of order milliwatts. The low efficiency is due to nonperfect simultaneous phase matching for the three processes since PM angle is wavelength-dependent. Furthermore, the use of the BPM technique leads to very stringent bandwidths and tolerances, so that angle or temperature tuning is required. This limits the use of BPM for simultaneous RGB generation.

\section{RGB generation by multiple quasi-phase-matching}

The above mentioned limitations can be overcome by using the quasi-phase matching (QPM) technique. The widely used material for QPM is a ferroelectric crystal with engineered domain structures. Here the reciprocal vectors provided by the domain structure plays an important role. The momentum conservation is modified into

$$
k_{2}-2 k_{1}=\mathrm{G}
$$

which is the so-called quasi-phase matching. Here $\mathrm{G}$ is a reciprocal vector of the domain structure.

With an aperiodic domain distribution, it is possible to obtain simultaneously different frequency conversion processes. ${ }^{8-10)} \mathrm{Si}$ multaneous generation of red, green, and blue continuous-wave laser radiation in a $\mathrm{Nd}^{3+}$-doped aperiodically poled lithiumniobate crystal $\left(\mathrm{Nd}^{3+}\right.$ :APPLN) has been demonstrated by Capmany. ${ }^{10)} \mathrm{Nd}^{3+}$ :APPLN congruent crystal was grown by the off-centered Czochralski method. That is, the growth axis was displaced away from the symmetry axis of the temperature field in the melt. The aperiodically poled sample was obtained accidentally during the crystal growth process. The bulk crystal was grown along the a axis. The $\mathrm{LiNbO}_{3}$ crystal used was a 3-mmlong crystal containing a $0.7 \mathrm{~mol} \%$ concentration of $\mathrm{Nd}^{3+}$ ions and having a cross-sectional input area of $4 \times 4 \mathrm{~mm}$.

The nonlinear laser crystal was end-pumped at $744 \mathrm{~nm}$. The system was specially designed so that the 1084 and $1372 \mathrm{~nm}$ fundamental waves oscillate simultaneously. The laser was operated with a total output power of $230 \mathrm{~mW}$ from $800 \mathrm{~mW}$ of absorbed pump power, and had an absorbed pump power of 52 $\mathrm{mW}$ at threshold. A chirp in the domain distribution provides a detuning curve for quasi-phase matching with a wavevector mismatch bandwidth wide enough to observe several intracavity self-frequency-conversion processes simultaneously. Red and green radiations were generated by SFD of the fundamental beam at $1084 \mathrm{~nm}$ and $1372 \mathrm{~nm}$, respectively. Blue light was generated by SFM of pump and $1084 \mathrm{~nm}$ laser radiation. The total visible output power was of order $1 \mathrm{~mW}$.

Continuous-wave red, green, and blue coherent light has also been simultaneously generated by quasi-phase matching in a strontium barium niobate crystal used as an intracavity frequency converter of a Nd: $\mathrm{YVO}_{4}$ laser operating at $1.34 \mu \mathrm{m} .{ }^{11-13)} \mathrm{SBN}$ is a solid solution with a tetragonal tungsten bronze structure, widely used as a photorefractive material due to its high electrooptic and nonlinear coefficients. When this ferroelectric crystal is cooled through its Curie transition temperature (without a pooling field), numerous domains are randomly, homogeneously and spontaneously generated to minimize the internal free energy. The domains in SBN crystals have a cylindrical shape (needlelike) with the longest dimension parallel to the optical axis and are randomly distributed in the crystal. The broad distribution of ferroelectric domain sizes $(1-8 \mu \mathrm{m})$ provides the possibility of different simultaneous frequency conversion processes without requiring angle or temperature tuning. Blue and red colors were generated by SHG of pump $(0.88 \mu \mathrm{m})$ and infrared $(1.34$ $\mu \mathrm{m})$ laser radiation, respectively. Green light was produced by sum frequency mixing of pump and laser radiations. In contrast to the case of $\mathrm{Nd}^{3+}$ :APPLN, where the generated RGB lights are collinear with the pump light, here visible radiation propagates in all directions perpendicular to the $c$ axis of the SBN crystal. Due to this dispersed nature of the visible radiation the output power could not be easily measured. An overall RGB power is estimated to be of about $1.5 \mathrm{~mW}$ for a pump power of $800 \mathrm{~mW}$.

\section{RGB generation by coupled quasi-phase-matching}

We have demonstrated the simultaneous generation of multiple-wavelength outputs by using engineered domains (so-called optical superlattice) with quasi-periodic, aperiodic even with periodic structures. ${ }^{14-16)}$ Usually QPM method is used with periodic structure, in which the selections of period and matching temperature are rather restricted. In fact, QPM can be extended from a periodic to a quasi-periodic structure. We demonstrated simultaneous SHG and direct THG in a Fibonacci structure. Here $\mathrm{TH}$ is generated not by the third-order nonlinear optical process but by the second-order nonlinear optical process. That is, TH is generated by a coupling of SHG process with sum frequency mixing process, both are of the second-order nature. ${ }^{17)}$ That's why we call it coupled quasi-phase matching.

However, for quasi-periodic structure, the locations of the reciprocals are not independent and are all related to an irrational number intrinsic to the structure (for Fibonacci structure, this number is the golden mean). Therefore, quasi-periodic structures rely on coincidences. Thus it is difficult, if not impossible, to realize multiply QPM coupled parametric processes at an arbitrary wavelength in the transparent range of the material.

We reported a different scheme for RGB light generation in a specially engineered domain structure. Here a $\mathrm{Nd}: \mathrm{GdVO}_{4}$ laser is used as a pump source, which operates at both 1342 and 1063 $\mathrm{nm}$ wavelengths. The RGB light output is realized by simultaneous frequency doubling and tripling of the fundamentals.

The Nd: $\mathrm{GdVO}_{4}$ laser has been reported as an excellent laser crystal in the past. $\mathrm{GdVO}_{4}$ belongs to the same group of oxide compounds as $\mathrm{YVO}_{4}$, crystallizing in a zircon structure with a tetragonal space group. Compared with $\mathrm{Nd}: \mathrm{YVO}_{4}, \mathrm{Nd}: \mathrm{GdVO}_{4}$ crystals have almost entirely similar lasing properties, but a much higher absorption coefficient and larger absorption cross-section. Besides this, $\mathrm{Nd}: \mathrm{GdVO}_{4}$ crystal is characterized by its unexpectedly high thermal conductivity along the 110] directions, which was measured to be comparable to that of Nd:YAG. ${ }^{16)}$

For QPM frequency conversion, the reciprocals play an important role. Usually the reciprocals can be obtained by Fourier transformation of a given microstructure. Alternatively, we can 
reverse the process, i.e., construct a microstructure from the given reciprocal vectors. Here in this work, RGB light generation involves one SFG and two SHG process. That is, three reciprocals are needed:

$$
\begin{aligned}
& k_{\mathrm{s} 1}-2 k_{\mathrm{f} 1}-G_{\mathrm{R}}=0 \\
& k_{\mathrm{s} 2}-2 k_{\mathrm{f} 2}-G_{\mathrm{G}}=0 \\
& k_{\mathrm{t} 1}-k_{\mathrm{s} 1}-k_{\mathrm{f} 1}-G_{\mathrm{B}}=0
\end{aligned}
$$

where $k$ is a wavevector and $G$ is a reciprocal vector, the subscripts $\mathrm{f}, \mathrm{s}$ and $\mathrm{t}$ indicate the fundamental, second harmonic and third harmonic, respectively. The subscripts 1 and 2 differentiate the processes based on the pump waves of 1342 and 1063 nm.

Based on the three reciprocals required $\left(G_{i}, i=R, G, B\right)$, a straightforward way to design is to use the following function:

$$
\mathrm{f}(x)=\sin \left(G_{\mathrm{R}} x\right)+a \sin \left(G_{\mathrm{G}} x\right)+b \sin \left(G_{\mathrm{B}} x\right)
$$

and

$$
\mathrm{g}(x)=\operatorname{sgn}[\mathrm{f}(x)]
$$

where sgn represents the signum function, corresponding to positive or negative domains. This is just the reverse Fourier transformation. The positions of the domain walls of the superlattice are determined by the roots of $\mathrm{f}(x)$. This function provides three reciprocals automatically. The coefficients $a$ and $b$ can be tuned to optimize the structure of the superlattice. When the ratio between any two of the three $G_{\mathrm{s}}$ is a rational number, then the structure becomes a periodic one.

In the designing, considering the limitation of the poling technique, the narrowest domains are controlled to be no less than 3 $\mu \mathrm{m}$. In other words, the domains whose lengths are less than 3 $\mu \mathrm{m}$ will flip their sign and merge into the adjacent domains, until all the domains are not shorter than $3 \mu \mathrm{m}$. In this way and setting the matching temperature to be at $110{ }^{\circ} \mathrm{C}$, we get the required structure. The Fourier spectrum of the designed structure is somewhat different from equation (6). Figure 1 shows the result. Apart from the three required components, there are many others. Since they do not satisfy QPM conditions, they do not participate in the nonlinear optical interactions. The Fourier coefficients of the three required reciprocals are $0.1851,0.4587$ and 0.2187 , respectively, which are shown in Fig. 1. These val-

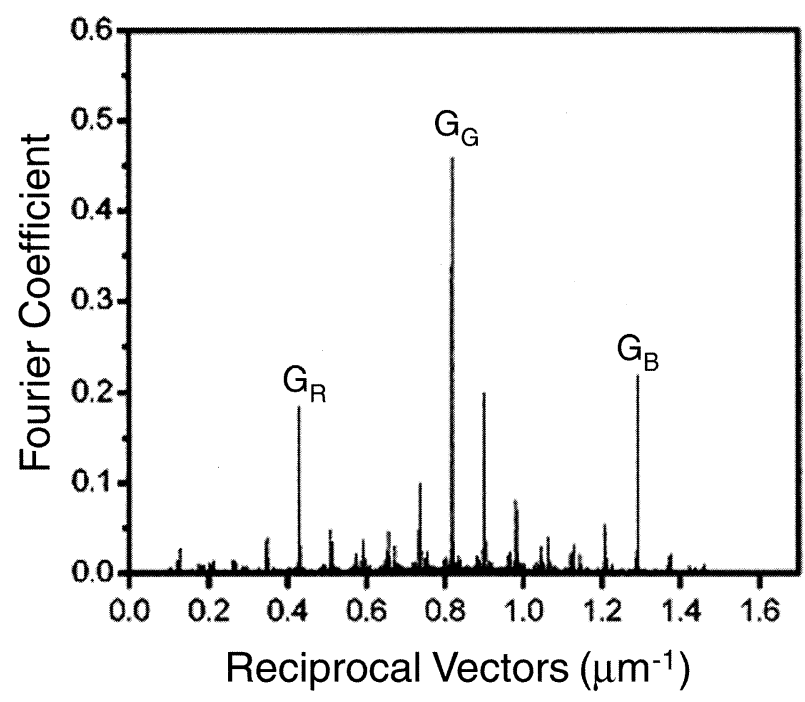

Fig. 1 The Fourier spectrum of the designed aperiodic OSL. ues are fairly large and can be adjusted independently. These are the obvious two advantages of this design scheme.

The superlattice made of a $\mathrm{LiTaO}_{3}$ crystal wafer was fabricated by a standard electric field poling technique at room temperature, with the pattern designed according to the above method. The samplewas observed after poling and etching and the size of the shortest domains was $2.8 \mu \mathrm{m}$. The domain walls show some irregularity, which would influence the conversion efficiency. The thickness of the wafer was about $0.5 \mathrm{~mm}$ and the length was $1.2 \mathrm{~cm}$. The two end-faces of the wafer were polished for optical measurement, but not coated.

The schematic diagram of our experimental measurement is shown as Fig. 2. The fundamentals of 1342 and $1063 \mathrm{~nm}$ were generated from a $Q$-switched $\mathrm{Nd}: \mathrm{GdVO}_{4}$ laser pumped by a fibre-coupled continuous diode laser (FAP-System, Coherent Inc.) at $808 \mathrm{~nm}$. The pumping output power was $15 \mathrm{~W}$ and the optical fibre diameter was $100 \mu \mathrm{m}$. The $4 \mathrm{~mm} \times 4 \mathrm{~mm} \times 7.6 \mathrm{~mm}$ laser host was a $\mathrm{Nd}: \mathrm{GdVO}_{4}$ crystal, whose dopant concentration, of $\mathrm{Nd}^{3+}$, is $0.5 \%$. M1 and M2 made up a two-mirror resonator with the length of $12.5 \mathrm{~cm}$. The input mirror M1 had antireflective (AR) coating at $808 \mathrm{~nm}$ on its entrance face and the high reflectivity (HR) coating at both 1342 and $1063 \mathrm{~nm}$ and high transmission (HT) coating at $808 \mathrm{~nm}$ on the other face. The beam sizes inside the laser host were estimated to be $\sim 377$ $\mu \mathrm{m}$ at $1342 \mathrm{~nm}$ and $~ 322 \mu \mathrm{m}$ at $1063 \mathrm{~nm}$. The output coupler M2 was a flat mirror with partial reflection (PR) coating at both $1342 \mathrm{~nm}(\mathrm{R}=96 \%)$ and $1063 \mathrm{~nm}(\mathrm{R}=35 \%)$ on one face and AR coating at both 1342 and $1063 \mathrm{~nm}$ on its other face. An acousto-optic $Q$-switch with a repetition rate of $10 \mathrm{kHz}$ is mounted inside the resonator cavity. For $1063 \mathrm{~nm}$ output, the pulse width was $23 \mathrm{~ns}$ and the average power was $580 \mathrm{~mW}$. The corresponding parameters for $1342 \mathrm{~nm}$ were $48 \mathrm{~ns}$ and $1.21 \mathrm{~W}$. The beam was focused by a lens $(f=25 \mathrm{~mm})$ and coupled into the polished entrance face of the sample. The radius of the beam waist inside the superlattice was about $45 \mu \mathrm{m}$. The beam quality factors (M2) were about 3.2 and 2.5 at 1063 and $1342 \mathrm{~nm}$, respectively. The sample was heated in an oven (model OTCPPLN-20, Super Optronics Ltd) in order to adjust the phasematching temperature with accuracy of $0.1{ }^{\circ} \mathrm{C}$. At the appropriate temperature, the RGB lights can be detected simultaneously.

Figure 3 shows the experimental results for RGB light output powers as a function of temperature. The measured phase-matching temperatures for red, green and blue lights were 122.5, 106.5 and $113.4{ }^{\circ} \mathrm{C}$, with the bandwidths (full width at half-maximum) of $3.5,11.2,1.5^{\circ} \mathrm{C}$ respectively. The maximum average powers were $251.5,193.3$ and $13.1 \mathrm{~mW}$, with conversion efficiencies of $20.8 \%, 33.3 \%$ and $1.1 \%$. (Here the conversion efficiency is defined to be the ratio of the output power of one colour to the input power at 1342 and $1063 \mathrm{~nm}$.). In the temperature tuning curves, the peaks of the three lights deviated from the expected

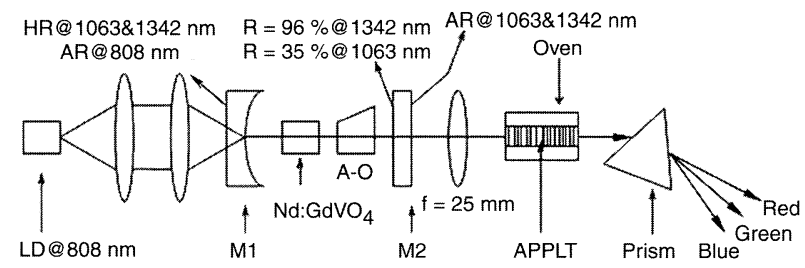

Fig. 2 Schematic of the experimental setup for the simultaneous generation of RGB. 


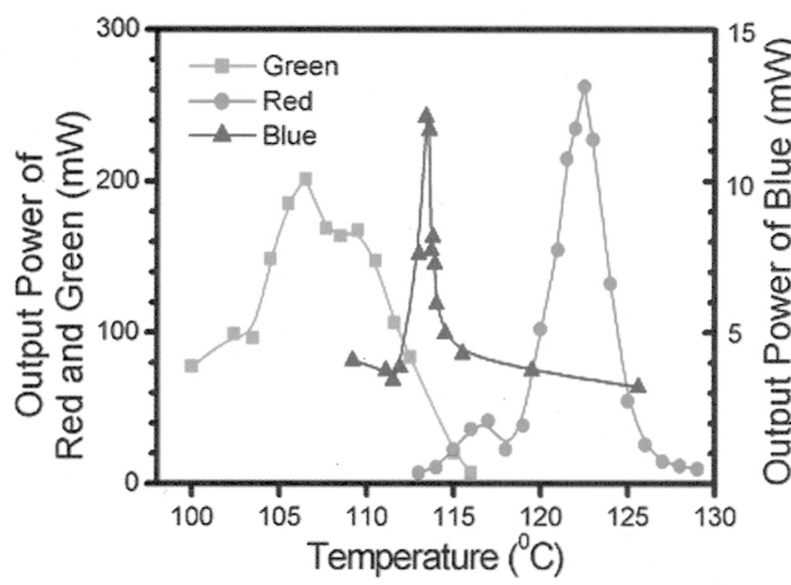

Fig. 3 The measured temperature tuning curves of the RGB outputs.

point due to some unsolved problems. The power levels and the conversion efficiencies were fairly high. At $115.8^{\circ} \mathrm{C}$, the RGB lights were generated simultaneously with $31.3,18.4$ and 3.7 $\mathrm{mW}$, respectively. Figure 4 shows the simultaneous output of RGB lights separated by a prism. However, the simultaneous oscillation at 1 and $1.3 \mathrm{~mm}$ in $\mathrm{Nd}^{3+}$ doped lasers requires a skilled design of cavity mirrors and pump geometry, and usually leads to fluctuations in laser power due to mode competition.

Simultaneous generation of GRB lights has also been realized in a $\mathrm{LiTaO}_{3}$ crystal with two periodic optical superlattices in series. Using a $532 \mathrm{~nm}$ laser as pump, the red light was generated at $631 \mathrm{~nm}$ as the signal of the optical parametric process in the first superlattice, and the blue light at $460 \mathrm{~nm}$ was the sum-frequency mixing the idler and the pump in the second superlattice. Along with the remnant pump at $532 \mathrm{~nm}, \mathrm{GRB}$ lights can be obtained from the superlattice simultaneously.

\section{Conclusion}

In the past, many attempts at simultaneous generation of RGB light have been reported. A brief review has been given in this field. In the as-grown crystals of $\mathrm{LiNbO}_{3}$ and $\mathrm{SBN}$, the domain structures are formed spontaneously during the crystal growth process, resulting in very low conversion efficiency. Our study showed that with a careful design of the domain engineering, RGB light can be realized by coupled quasi-phase matching method. The potential high efficiency and multiplex output wavelength make this scheme emulous to realize a compact GRB laser.

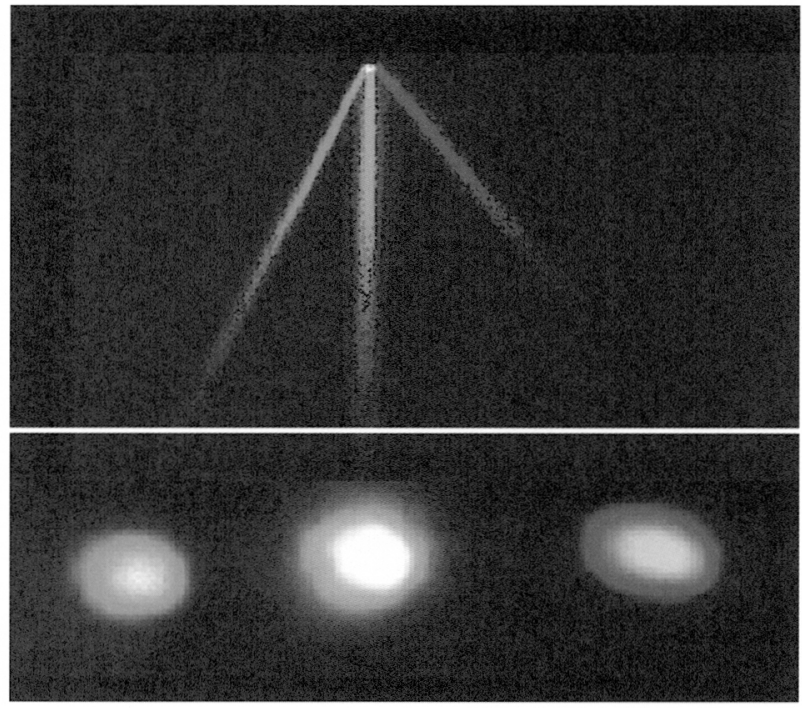

Fig. 4 Potograph of RGB beam paths and beam spots.

\section{References}

1) J. Wallacè: Laser Focus World 35 (1999) 15.

2) P. Baldi, C. G. Trevifio-Palacios, G. I. Stegeman, M. P. De Micheli, D. B. Ostrowsky, D. Delacourt, and M. Papuchon: Electron. Lett. 31 (1995) 1350.

3) E. Cantelar, G. A. Torchia, J. A. Sanz-Garcia, P. L. Pernas, G. Lifante, and F. Cusso: Appl. Phys. Lett. 83 (2003) 2991.

4) S. S. Yap, W. O. Siew, T. Y. Tou, and S. W. Ng: Appl. Opt. 41 (2002) 1725.

5) D. Jaque, J. Capmany, and J. Garcia Sole: Opt. Eng. 38 (1999) 1794. 6) D. Japue, J. Capamny, and J. Garcia Sole: Appl. Phys. Lett. 75 (1999) 325.

7) D. Jaque: J. Alloys and Compounds 323-324 (2001) 204.

8) J. Capmany, V. Bermudez, D. Callejo, J. Garcia Sole, and E. Dieguez: Appl. Phys. Lett. 76 (2000) 1225.

9) J. Capmany, J. A. Pereda, V. Bermudez, D. Callejo, and E. Dieguez: Appl. Phys. Lett. 79 (2001) 1751.

10) J. Capmany: Appl. Phys. Lett. 78 (2001) 144.

11) J. J. Romero, D. Jaque, J. G. Sole, and A. A. Kaminskii: Appl. Phys. Lett. 78 (2001) 1961.

12) J. J. Romero, D. Jaque, J. Garcia Sole, and A. A. Kaminskii: Appl. Phys. Lett. 81 (2002) 4106.

13) D. Jaque and J. J. Romero: Optical Materials 24 (2003) 411.

14) Z. W. Liu, S. N. Zhu, Y. Y. Zhu, H. Liu, Y. Q. Lu, W. T. Wang, N. B. Ming, X. Y. Liang, and Z. Y. Xu: Solid State Comm. 119 (2001) 363.

15) J. Liao, J. L. He, H. Liu, H. T. Wang, S. N. Zhu, Y. Y. Zhu, and N. B. Ming: Appl. Phys. Lett. 82 (2003) 3159.

16) T. W. Ren, J. L. He, C. Zhang, S. N. Zhu, Y. Y. Zhu, and Y. Hang: J. Phys. Condens. Matter 16 (2004) 3289.

17) C. Zhang, Y. Y. Zhu, S. X. Yang, Y. Q. Qin, S. N. Zhu, Y. B. Chen, H. Liu, and N. B. Ming: Opt. Letters 25 (2000) 436. 